\title{
KHẢO SÁT CÁC NỀn TẢNG VÀ KỸ THUẠT XỦ LÝ LOG TRUY CẬP DỊCH VỤ MẠNG CHO PHÁT HIỆN NGUY CƠ MẤT AN TOÀN THÔNG TIN
}

\author{
Phạm Duy Lộca ${ }^{a^{*}}$ Hoàng Xuân Dậu \\ ${ }^{a}$ Khoa Công nghệ Thông tin, Truờng Đại học Đà Lạt, Lâm Đồng, Việt Nam \\ ${ }^{b}$ Khoa Công nghệ Thông tin, Học viện Công nghệ Buu chính Viễn thông, Hà Nội, Việt Nam \\ "Tác giả liên hệ: Email: locpd@dlu.edu.vn \\ Lịch sử bài báo \\ Nhận ngày 15 tháng 01 năm 2018 \\ Chỉnh sửa ngày 19 tháng 05 năm 2018 | Chấp nhận đăng ngày 23 tháng 05 năm 2018
}

\section{Tóm tắt}

Trong hệ thống lớp các giải pháp đảm bảo an toàn thông tin, các giải pháp giám sát phát hiện bất thuoơng và các nguy cơ mất an toàn trong hệ thống mang được xem là lớp phòng vệ thứ hai, sau lớp tuoòng lưa và các biện pháp kiểm soát truy nhập. Lớp giải pháp này gồm các hệ thống giám sát, phát hiện và ngăn chặn tấn công, xâm nhập cho các host và mạng. Bài báo này khảo sát, đánh giá các nền tảng, công cu và các kỹ thuật xủ lý, phân tích log truy cập các máy chủ dịch vụ phục vu phát hiện các hành vi bất thường và nguy co mất an toàn thông tin. Trên cơ sở đó, bài báo đề xuất mô hình kiến trúc hệ thống giám sát, hố trợ đảm bảo an toàn thông tin cho các tổ chức có quy mô hệ thống mạng và nguồn lực hạn chế.

Từ khóa: Phát hiện bất thường; Phát hiện xâm nhập; Quản lý thông tin và sự cố an ninh.

Mã số định danh bài báo: http://tckh.dlu.edu.vn/index.php/tckhdhdl/article/view/405

Loại bài báo: Bài báo nghiên cứu gốc

Bản quyền @ 2018 (Các) Tác giả.

Cấp phép: Bài báo này được cấp phép theo CC BY-NC-ND 4.0 


\title{
A SURVEY OF NETWORK SERVICE LOG PROCESSING PLATFORMS AND TECHNIQUES FOR THE DETECTION OF INFORMATION INSECURITY RISKS
}

\author{
Pham Duy Loc ${ }^{a^{*}}$, Hoang Xuan Dau ${ }^{\mathrm{b}}$ \\ ${ }^{a}$ The Faculty of Information Technology, Dalat University, Lamdong, Vietnam \\ ${ }^{b}$ The Faculty of Information Technology, Posts and Telecommunications Institute of Technology, \\ Hanoi, Vietnam \\ ${ }^{*}$ Corresponding author: Email: locpd@dlu.edu.vn
}

Article history

Received: January $15^{\text {th }}, 2018$

Received in revised form: May 19 ${ }^{\text {th }}, 2018 \mid$ Accepted: May $23^{\text {rd }}, 2018$

\begin{abstract}
In the layers of information security measures, the monitoring and detection measures of anomalous activities and information insecurity risks are considered the second defense layer behind firewalls and access controls. This defense layer includes intrusion detection and prevention systems for hosts and networks. This paper examines platforms, tools and techniques for processing and analyzing access logs of network service servers for the detection of anomalous activities and information insecurity risks. Based on the survey results, the paper proposes the architecture of the monitoring and information security ensurance system for small and medium-sized networks of organizations with limited resources.
\end{abstract}

Keywords: Anomaly detection; Intrusion detection; Security information and event management.

Article identifier: http://tckh.dlu.edu.vn/index.php/tckhdhdl/article/view/405

Article type: (peer-reviewed) Full-length research article

Copyright (c) 2018 The author(s).

Licensing: This article is licensed under a CC BY-NC-ND 4.0 


\section{1. ĐẶT VẤN Đề}

Việc giám sát thu thập, xử lý và phân tích các nhật ký, hay vết truy cập (access $\log$, từ đây gọi tắt là $\log$ ) mạng nói chung và các log truy cập các dịch vụ mạng nói riêng là nhiệm vụ không thể thiếu trong các hệ thống giám sát, phát hiện bất thường, phát hiện tấn công, xâm nhập hệ thống và mạng. Từ dữ liệu log thô thu thập được, qua quá trình xử lý, phân tích, chúng ta có thể trích xuất được các thông tin quan trọng về dấu hiệu, hoặc khả năng xuất hiện của các hành vi truy cập bất thường, các dạng mã độc và các dạng tấn công, xâm nhập. Kết quả phân tích, phát hiện dấu hiệu xuất hiện của các truy cập bất thường, mã độc, tấn công, xâm nhập là đầu vào quyết định việc đưa ra các cảnh báo nguy cơ mất an toàn thông tin đối với hệ thống. Đồng thời, kết quả phân tích, phát hiện cũng là một trong các căn cứ quan trọng hỗ trợ việc đánh giá, lựa chọn và triển khai các giải pháp đảm bảo an toàn phù hợp cho thông tin, hệ thống và các tài nguyên mạng. Vấn đề này càng quan trọng hơn trong bối cảnh an toàn thông tin ở Việt Nam đã và đang trở thành vấn đề nóng được các cơ quan, tổ chức chính phủ, các doanh nghiệp và cả xã hội quan tâm.

Theo Báo cáo An toàn thông tin Việt Nam 2016 (Cục An toàn Thông tin, 2016) thì tình hình an toàn thông tin Việt Nam năm 2016 tiếp tục diễn biến phức tạp. Theo đó, số lượng các cuộc tấn công mang có chiều hướng tăng so với các năm trước đây, nhất là các tấn công mạng vào hệ thống thông tin của cơ quan nhà nước và các doanh nghiệp lớn. Tấn công mạng tiếp tục tăng về quy mô và số lượng, nhất là các cuộc tấn công từ chối dịch vụ phân tán (Distributed Denial of Service - DDoS) và tấn công có chủ đích (Advanced Persistent Threats - APT). Điển hình là vụ tấn công mạng vào Tổng Công ty Hàng không Việt Nam vào cuối tháng 7 năm 2016. Nhiều cơ quan, tổ chức khác của nhà nước sau khi được rà soát, kiểm tra, đánh giá an toàn cho hệ thống thông tin cũng đã phát hiện ra nhiều điểm yếu, lỗ hổng, bị lây nhiễm phần mềm độc hại và nguy cơ mất an toàn thông tin là rất lớn.

Báo cáo An toàn thông tin Việt Nam 2016 (Cục An toàn Thông tin, 2016) cũng dự báo tình hình an toàn thông tin Việt Nam trong năm 2017 và những năm tới sẽ tiếp tục diễn biến phức tạp với sự bùng nổ của các thiết bị IoT và sự phát triển mạnh của các dịch vụ trực tuyến trên nền $\mathrm{Web} /$ Internet. Do vậy, yêu cầu đảm bảo an toàn cho hệ thống thông tin của các cơ quan tổ chức chính phủ, doanh nghiệp và đảm bảo tính riêng tư của từng người dùng là cấp thiết. Với hệ thống máy chủ cung cấp dịch vụ mạng thiết yếu, như các máy chủ Web và các máy chủ tên miền, việc giám sát phát hiện các truy nhập bất thường và cảnh báo các nguy cơ mất an toàn thông tin là một nội dung đặc biệt quan trọng trong nhóm các giải pháp đảm bảo an toàn thông tin cho hệ thống cung cấp dịch vụ, đảm bảo cho hệ thống có thể hoạt động thông suốt, giảm thiểu thời gian ngừng dịch vụ do sự cố an toàn thông tin.

Bài báo này trước hết khảo sát, đánh giá các nền tảng, công cụ và các kỹ thuật xử lý, phân tích log truy cập phục vụ phát hiện các hành vi bất thường và nguy cơ mất an toàn thông tin trong các hệ thống cung cấp dịch vụ mạng, bao gồm các hệ thống dịch 
vụ Web và dịch vụ tên miền. Dựa trên kết quả khảo sát, đánh giá, bài báo đề xuất kiến trúc hệ thống giám sát, hỗ trợ đảm bảo an toàn thông tin cho các cơ quan, tổ chức có quy mô hệ thống mạng và nguồn lực hạn chế.

Phần còn lại của bài báo được bố cục như sau: Mục 2 khảo sát, so sánh các nền tảng và công cụ xử lý, phân tích log truy cập. Mục 3 phân tích các kỹ thuật phát hiện các nguy cơ mất an toàn thông tin dựa trên phân tích log truy cập. Mục 4 là phần nhận xét và đề xuất, và Mục 5 là kết luận của bài báo.

\section{CÁC NỀn TẢNG VÀ CÔNG CỤ XỦ LÝ, PHÂN TÍCH LOG TRUY CẬP}

Hiện nay, có nhiều nền tảng và công cụ xử lý, phân tích log truy cập thương mại cũng như mã mở được cung cấp như IBM QRadar SIEM, Splunk, Sumo Logic, VNCS Web Monitoring, Logstash, Graylog, LOGalyze, Webalizer... Mục này khảo sát chi tiết hơn về tính năng và các ưu nhược điểm của các nền tảng và công cụ kể trên, trong đó các tiêu chí quan trọng được xem xét bao gồm: (i) Hỗ trợ thu thập, xử lý các dạng log truy cập từ nhiều nguồn; (ii) Có khả năng phát hiện và cảnh báo các truy nhập bất thường và các nguy cơ mất an toàn thông tin, như các dạng tấn công, xâm nhập vào hệ thống được giám sát; (iii) Khả năng quản lý, lưu trữ, tìm kiếm log và tạo các dạng báo cáo, thống kê; và (vi) Chi phí cài đặt và vận hành.

\subsection{Các nền tảng xử lý log truy cập thương mại}

\subsubsection{IBM QRadar SIEM}

QRadar SIEM (Security Information and Event Management) là hệ thống quản lý các thông tin và sự cố an ninh được phát triển và cung cấp bởi hãng IBM, Hoa Kỳ. QRadar SIEM (IBM QRadar, 2017) cho phép phát hiện các bất thường, các nguy cơ với độ chính xác cao và tỷ lệ cảnh báo sai thấp thông qua việc xử lý, phân tích dữ liệu log và luồng mạng từ hàng ngàn thiết bị và ứng dụng phân tán trong mạng, như minh họa trên Hình 1. QRadar SIEM có thể được cài đặt tại hệ thống mạng của khách hàng, hoặc hoạt động như một dịch vụ SIEM trên nền điện toán đám mây. Các tính năng tiêu biểu của QRadar SIEM bao gồm (IBM QRadar, 2017):

- Cảm nhận, phát hiện giả mạo, các nguy cơ bên trong và bên ngoài;

- Thực hiện việc chuẩn hóa và tương quan các sự kiện tức thời;

- Cảm nhận, theo dõi và liên kết các sự cố và nguy cơ;

- Hỗ trợ cài đặt tại hệ thống mạng của khách hàng, hoặc có thể truy cập như một dịch vụ SIEM trên nền điện toán đám mây; 
- Có thể bổ sung dung lượng lưu trữ và năng lực xử lý nhanh chóng và rẻ tiền;

- Tích hợp tri thức phát hiện nguy cơ từ IBM X-Force;

- Hỗ trợ vấn đề quản lý và phối hợp phòng chống nguy cơ;

- Có khả năng tích hợp với các sản phẩm khác của hãng IBM, hoặc hãng khác.
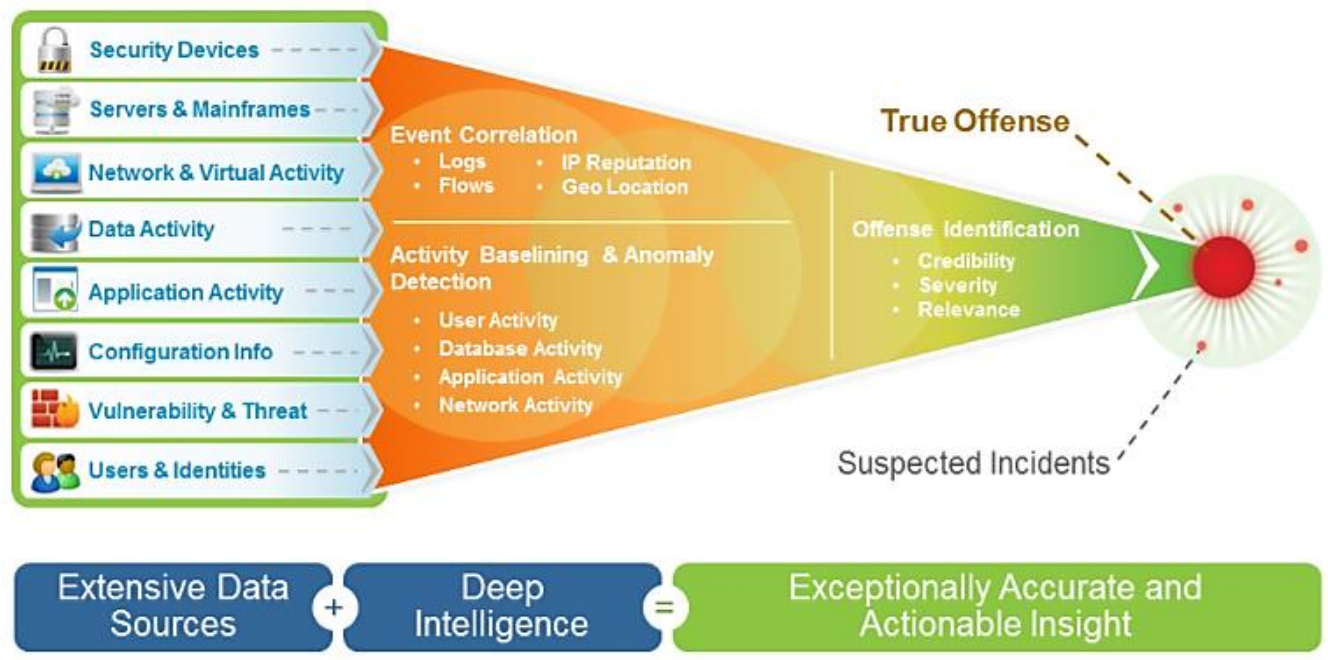

\section{Hình 1. Mô hình thu thập dữ liệu và xử lý của QRadar SIEM}

Nguồn: IBM QRadar (2017).

IBM QRadar SIEM đã được triển khai sử dụng và được đánh giá cao ở các cơ quan, tổ chức chính phủ, ngân hàng và doanh nghiệp có quy mô hệ thống mạng lớn. Tuy nhiên, hạn chế lớn nhất của QRadar SIEM là chi phí cài đặt ban đầu và phí bản quyền khá lớn, nên không thực sự thích hợp với các cơ quan, tổ chức có hệ thống mạng có quy mô vừa và nhỏ với nguồn lực hạn chế.

\subsubsection{Splunk}

Splunk (Splunk, 2017) là một nền tảng xử lý và phân tích log rất mạnh, được cung cấp bởi hãng Splunk Inc., Hoa Kỳ. Splunk có hàng trăm công cụ tích hợp, cho phép xử lý nhiều loại log khác nhau với khối lượng lớn theo thời gian thực. Splunk có thể xử lý, phân tích log phục vụ đảm bảo an toàn thông tin, cũng như trích rút thông tin hỗ trợ cho các hoạt động kinh doanh. Splunk cung cấp các công cụ tìm kiếm và biểu đồ cho phép biểu diến kết qua đầu ra theo nhiều dạng. Hình 2 biểu diễ̃n màn hình tổng hợp an ninh (Security Posture Dashboard) của Splunk. 
Splunk có ba phiên bản, bao gồm: Splunk Enterprise cho các khách hàng có nhu cầu xử lý log tại chỗ với khối lượng lớn; Splunk Cloud cho các khách hàng tải log lên nền tảng đám mây của Splunk để xử lý; và Splunk Light cho các khách hàng có nhu cầu xử lý log tại chỗ với khối lượng vừa và nhỏ. Hạn chế lớn nhất của Splunk là chi phí cài đặt lớn, do khoản đầu tư ban đầu cho hệ thống thiết bị chuyên dụng có độ phức tạp cao. Một vấn đề khác là phí bản quyền hàng năm của Splunk cũng rất đắt đỏ (ước tính có thể lên đến hàng chục ngàn đô-la Mỹ mỗi năm), nên Splunk không thực sự thích hợp với các cơ quan, tổ chức có hệ thống mạng có quy mô vừa và nhỏ với nguồn lực hạn chế.

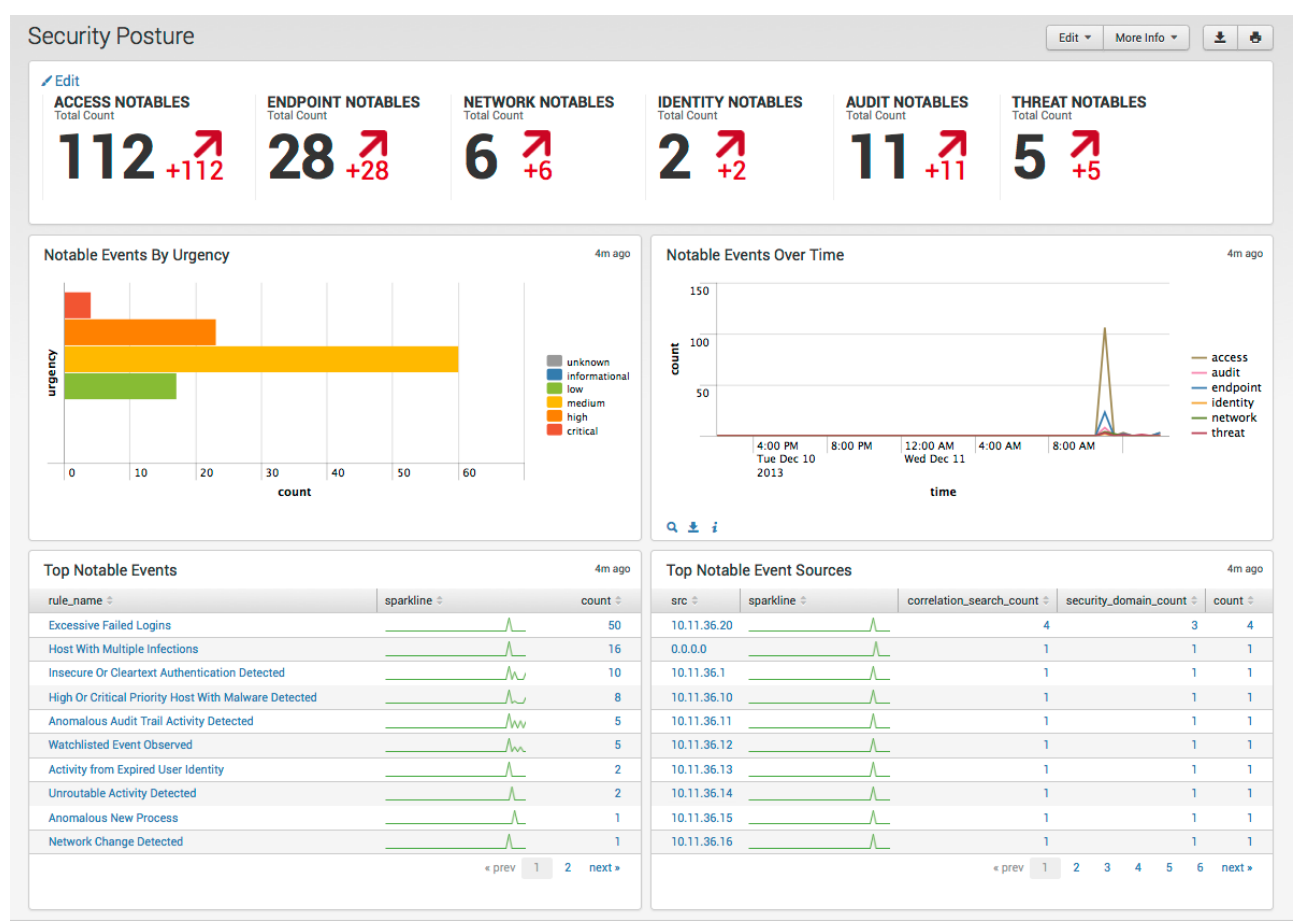

\section{Hình 2. Giao diện tổng hợp của Splunk}

Nguồn: Splunk (2017).

\subsubsection{Sumo Logic}

Sumo Logic (Sumo Logic, 2017) là một dịch vụ xử lý, phân tích và quản lý log trên nền tảng điện toán đám mây. Uu điểm của Sumo Logic là cung cấp nhiều tính năng và có khả năng xử lý nhiều loại log, đồng thời việc cài đặt cũng tương đối dễ dàng do Sumo Logic dựa trên nền tảng điện toán đám mây, không đòi hỏi thiết bị chuyên dụng. Hình 3 minh họa mô hình thu thập và xử lý log của Sumo Logic, theo đó log được thu thập từ hệ thống của khách hàng sử dụng các Agent/Collector và được tải lên hệ thống xử lý và phân tích của Sumo Logic.

Nhược điểm lớn nhất của Sumo Logic là việc phải tải khối lượng lớn log (có thể lên đến hàng chục GB/ngày) từ hệ thống sinh log lên hệ thống dịch vụ Sumo Logic để 
xử lý. Việc này đòi hỏi chi phí lớn cho đường truyền, có thể gây ra chậm trễ trong quá trình xử lý và tiềm ẩn nguy cơ rò rỉ dữ liệu nhạy cảm chứa trong log.

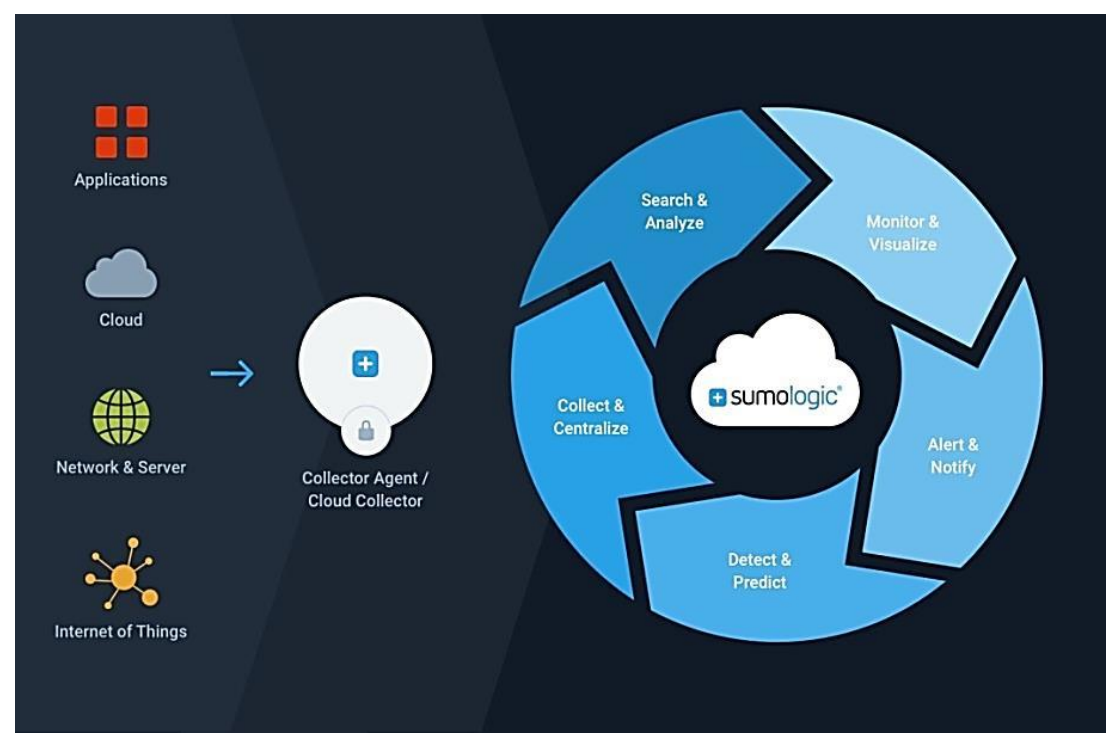

Hình 3. Mô hình thu thập và xử lý log của Sumo Logic

Nguồn: Sumo Logic (2017).

\subsubsection{VNCS Web Monitoring}

VNCS Web monitoring (VNCS, 2017) là giải pháp cho phép giám sát nhiều Website đồng thời dựa trên thu thập, xử lý và phân tích log truy cập sử dụng nền tảng Splunk do Công ty cổ phần Công nghệ An ninh không gian mạng Việt Nam phát triển. Hình 4 là một màn hình thống kê của VNCS Web monitoring.

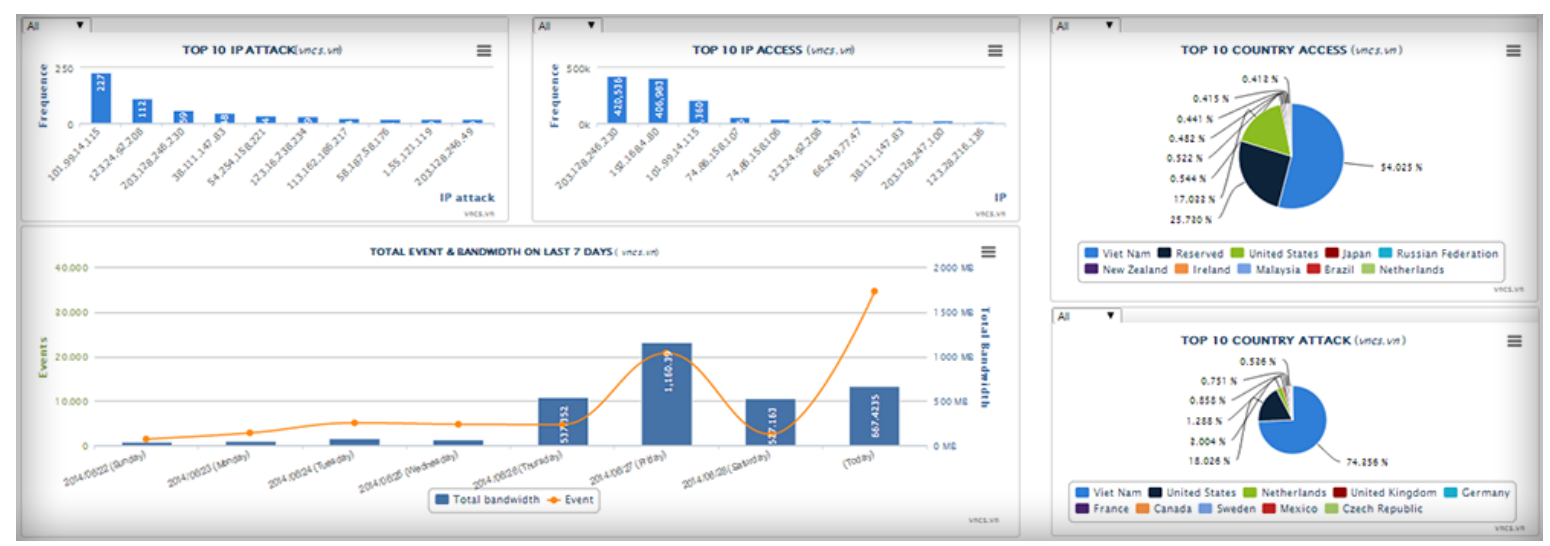

Hình 4. Một màn hình thống kê của VNCS Web monitoring

Nguồn: VNCS (2017). 
VNCS Web monitoring thu thập Web log từ các máy chủ cần giám sát, sau đó chuyển về hệ thống trung tâm để xử lý, phân tích. Hệ thống này cho phép quản lý log tập trung, hỗ trợ phân tích log thủ công để tìm sự cố, hỗ trợ giám sát và cảnh báo trạng thái hoạt động của Website, hỗ trợ phát hiện các dạng tấn công thay đổi nội dung, thay đổi giao diện, tấn công chèn mã SQL ( $S Q L$ Injection - SQLi), tấn công chèn mã script liên miền (Cross Site Scripting - XSS) và phát hiện mã độc trên Website. Hạn chế của VNCS Web monitoring là chỉ có khả năng xử lý và phân tích Web log.

\subsection{Các nền tảng mã mở}

\subsubsection{Logstash}

Logstash (Logstash, 2017) là một công cụ mã mở cho phép thu thập, xử lý và quản lý các file log. Logstash không hoạt động độc lập mà sử dụng công cụ ElasticSearch (https://www.elastic.co) để lập chỉ số và tìm kiếm dữ liệu, và công cụ Kibana (https://www.elastic.co/products/kibana) để biểu diễn dữ liệu dưới dạng biểu đồ. Hình 5 mô tả giao diện của Kibana hiển thị kết quả xử lý của Logstash. Uu điểm nổi bật của Logstash là mã mở và chi phí cài đặt và vận hành tương đối thấp. Tuy nhiên, nhược điểm của Logstash là phụ thuộc vào nhiều công cụ khác được phát triển bằng nhiều ngôn ngữ khác nhau dẫn đến khó khăn trong triển khai và bảo trì.

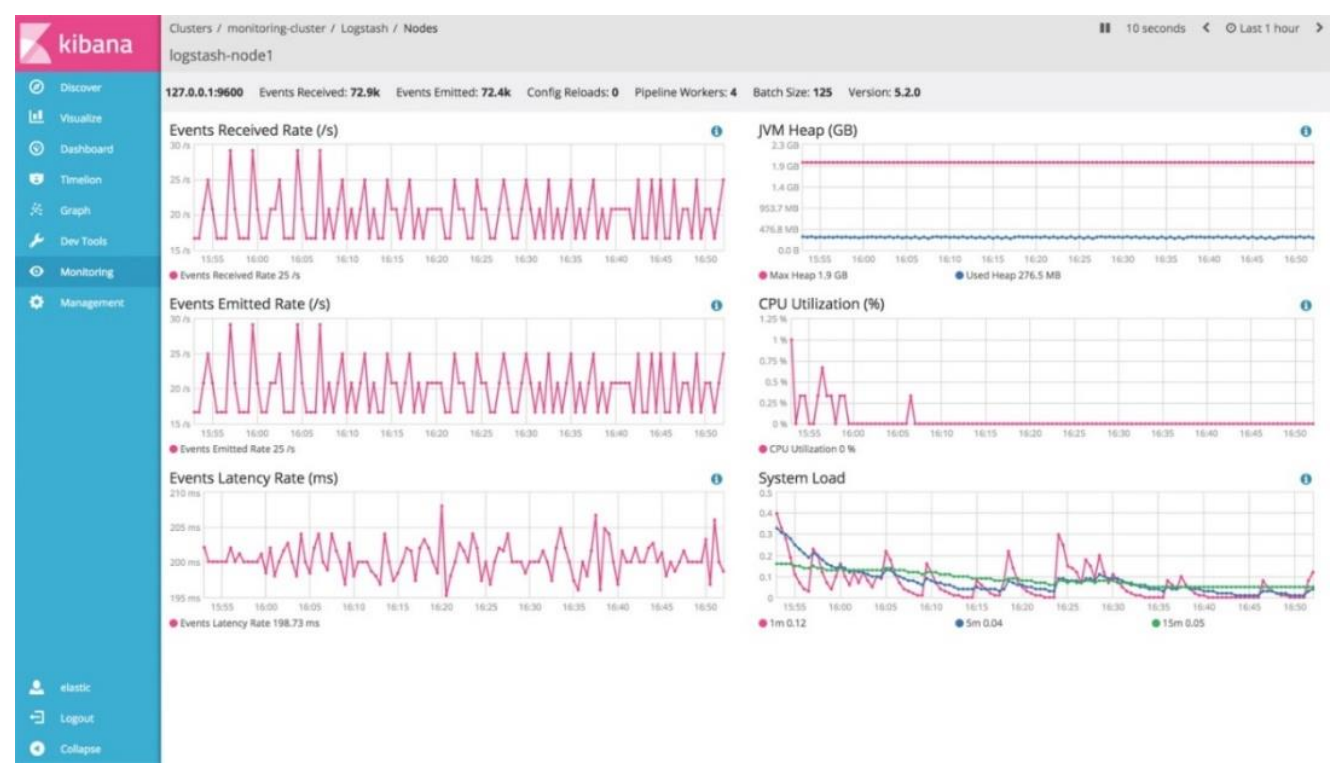

\section{Hình 5. Giao diện của Kibana hiển thị kết quả xử lý của Logstash}

\subsubsection{Graylog}

Graylog (Graylog, 2017) là một nền tảng mã mở cho phép xử lý, phân tích log truy cập từ nhiều nguồn theo thời gian thực. Graylog có khả năng phân tích hành vi người dùng, ứng dụng cho phát hiện và cảnh báo các truy cập bất thường cũng như trích 
xuất các mẫu hành vi truy cập phục vụ cho tối ưu hóa các trang Web. Graylog cũng cho phép ánh xạ từ ID sang tên truy nhập của người dùng và ánh xạ từ địa chỉ IP sang vị trí địa lý. Hình 6 minh họa màn hình tổng hợp của Graylog cho phép quản lý tập trung các tính năng xử lý và phân tích các nguồn log.

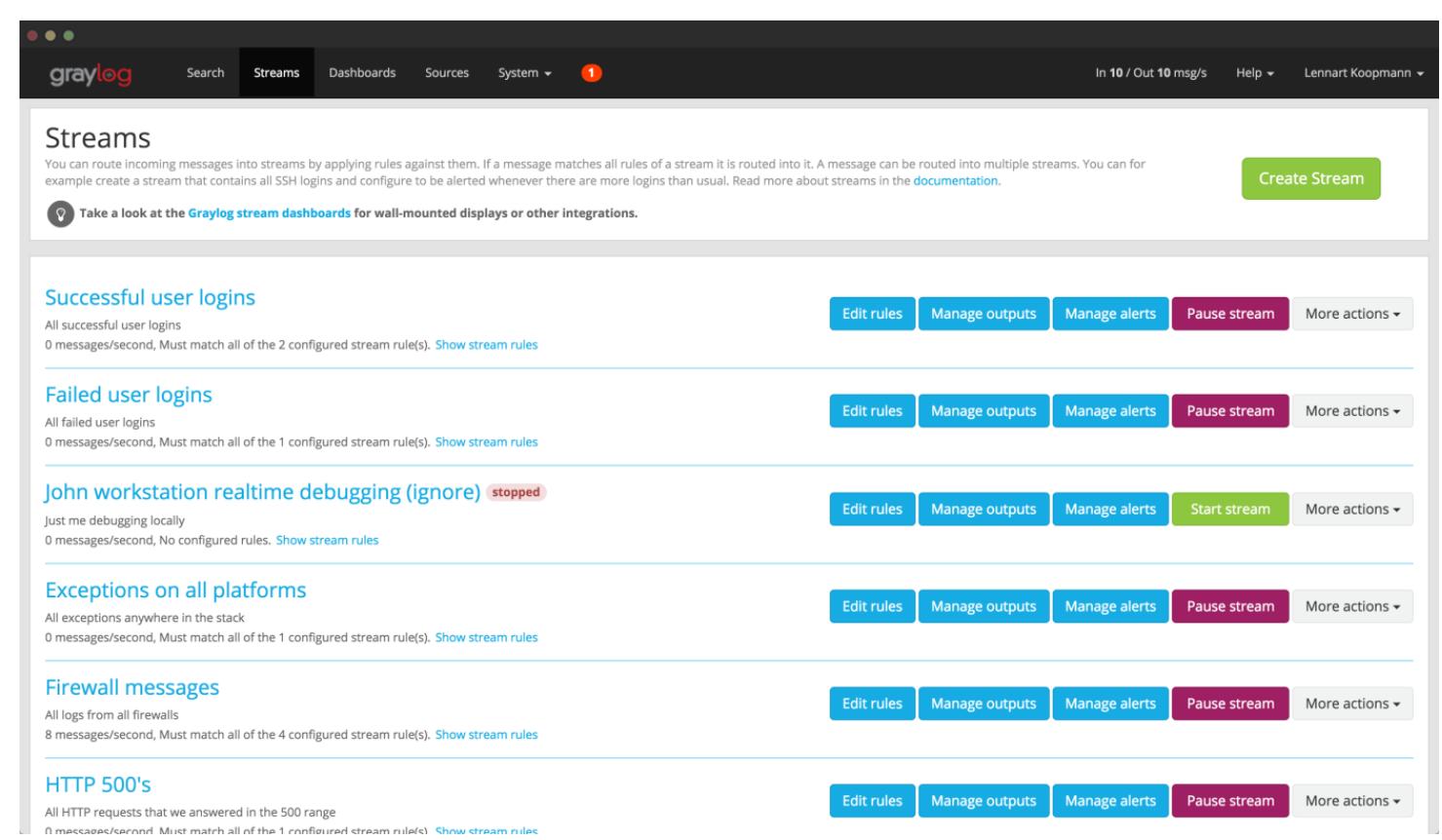

\section{Hình 6. Màn hình tổng hợp của Graylog}

Nguồn: Graylog (2017).

Mặc dù Graylog có khả năng nhận dạng các hành vi truy cập bất thường, nhưng nó không cho phép phân tích chuyên sâu các nguy cơ mất an toàn thông tin, như dấu hiệu xuất hiện các dạng mã độc và các dạng tấn công lên các dịch vụ và tài nguyên mạng.

\subsubsection{LOGalyze}

LOGalyze (LOGalyze, 2017) là một phần mềm mã nguồn mở cho phép quản lý $\log$ và giám sát mạng tập trung. LOGalyze hỗ trợ xử lý log từ nhiều nền tảng, bao gồm log từ các máy chủ Unix/Linux, Windows và các thiết bị mạng với khả năng xử lý, tìm kiểm và phát hiện các bất thường theo thời gian thực. LOGalyze còn cho phép người dùng định nghĩa các sự kiện và các cảnh báo dựa trên các dữ liệu log thu thập, xử lý. Hình 7 minh họa màn hình quản lý các dạng log của LOGalyze. Ngoài ra, LOGalyze còn là công cụ quản lý và giám sát mạng, hỗ trợ phát hiện mức truy cập bất thường và các sự cố mạng. Tuy nhiên, tương tự như Graylog, LOGalyze hầu như không có khả năng phân tích chuyên sâu các nguy cơ mất an toàn thông tin, như dấu hiệu xuất hiện các dạng mã độc và các dạng tấn công lên các dịch vụ và tài nguyên mạng. 


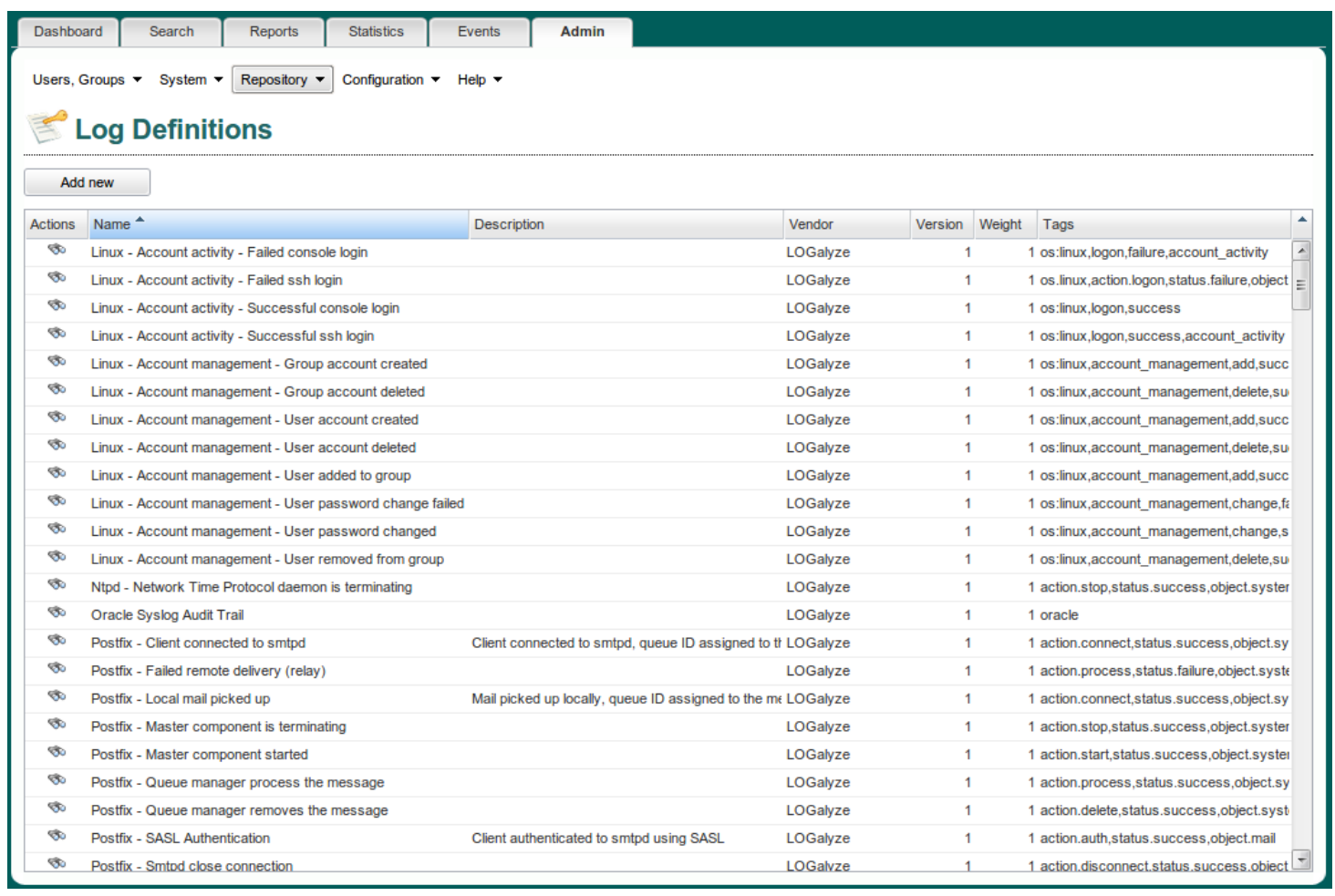

\section{Hình 7. Màn hình quản lý các dạng log của LOGalyze}

Nguồn: LOGalyze (2017).

\subsubsection{Webalizer}

Webalizer (Webalizer, 2017) là công cụ mã nguồn mở cho phép xử lý và phân tích log cho các Website. Webalizer là một trong các công cụ được sử dụng rộng rãi nhất cho quản trị máy chủ Web. Webalizer có khả năng phân tích các dạng log của các trang Web và tạo ra các báo cáo sử dụng các trang Web của người dùng, bao gồm lưu lượng truy nhập, các trang tham chiếu, phân bố người dùng theo vị trí địa lý và lượng dữ liệu tải xuống. Hình 8 minh họa một báo cáo thống kê về truy nhập trang Web theo tháng của Webalizer. Uu điểm của Webalizer là hỗ trợ phân tích nhiều dạng Web log và tạo được các báo cáo với các biểu đồ có tính biểu diễn cao. Tuy nhiên, Webalizer chỉ có khả năng phân tích tình hình sử dụng các trang Web mà ít có khả năng trích xuất các thông tin phục vụ cho việc cảnh báo các nguy cơ mất an toàn thông tin. 


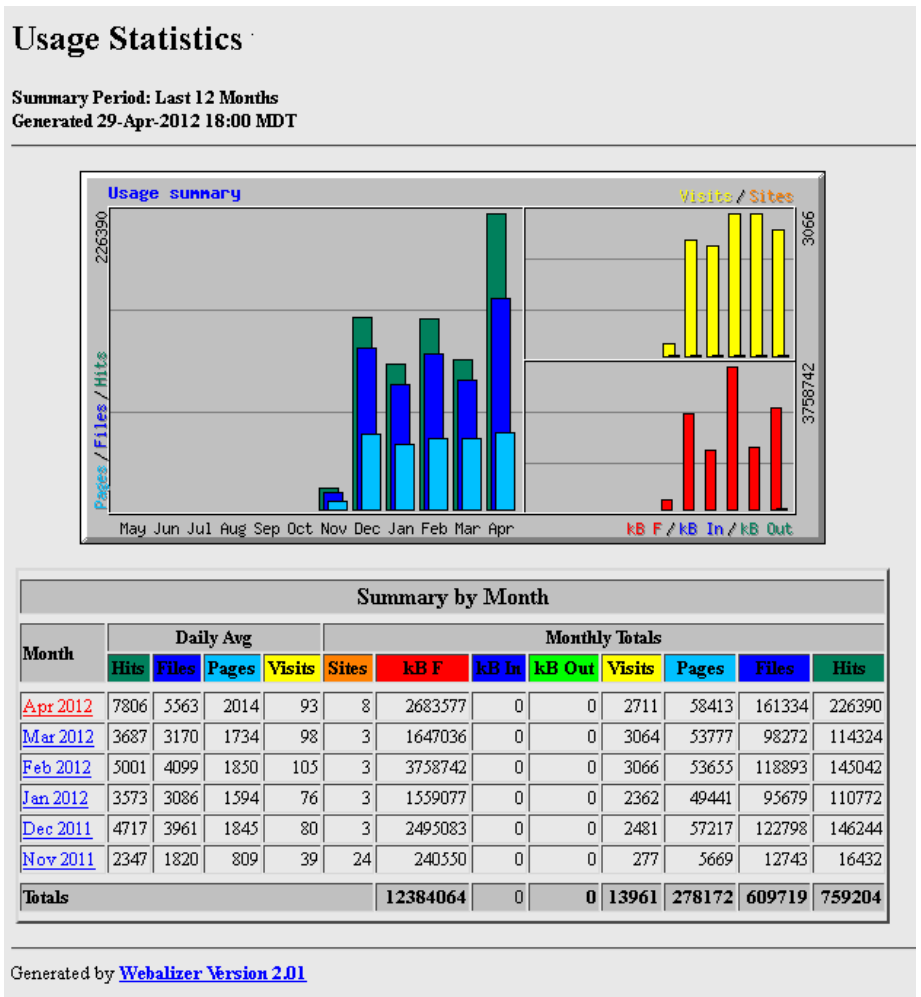

\section{Hình 8. Một mẫu báo cáo của Webalizer}

Nguồn: Webalizer (2017).

\subsection{So sánh các nền tảng và công cụ xử lý log truy cập}

Bảng 1 cung cấp thông tin so sánh các ưu điểm và nhược điểm của các nền tảng, công cụ xử lý, phân tích log truy cập ở trong và ngoài nước.

\section{Bảng 1. So sánh các nền tảng và công cụ xử lý log truy cập}

\begin{tabular}{|c|c|c|}
\hline Nền tảng & U’u điểm & Nhược điểm \\
\hline \multirow{3}{*}{$\begin{array}{l}\text { IBM } \\
\text { QRadar } \\
\text { SIEM }\end{array}$} & $\begin{array}{l}\text { - Hỗ trợ thu thập và xử lý nhiều loại log khác nhau } \\
\text { với khối lượng lớn và dữ liệu từ luồng mạng }\end{array}$ & $\begin{array}{l}\text { - Chi phí cài đặt ban đầu và phí bản } \\
\text { quyền khá lớn }\end{array}$ \\
\hline & - Hỗ trợ thu thập dữ liệu từ hàng ngàn thiết bị mạng & - Đòi hỏi thiết bị chuyên dụng \\
\hline & $\begin{array}{l}\text { - Hỗ trợ phát hiện các bất thường, các nguy cơ ATTT } \\
\text { với độ chính xác cao và tỷ lệ cảnh báo sai thấp }\end{array}$ & - Khó khăn trong vận hành và bảo trì. \\
\hline \multirow[t]{4}{*}{ Splunk } & $\begin{array}{l}\text { - Hỗ trợ xử lý nhiều loại log khác nhau với khối } \\
\text { lượng lớn theo thời gian thực }\end{array}$ & $\begin{array}{l}\text { - Chi phí bản quyền, cài đặt và vận } \\
\text { hành cao }\end{array}$ \\
\hline & - Hỗ trợ phân tích log phục vụ đảm bảo an toàn & - Đòi hỏi thiết bị chuyên dụng \\
\hline & $\begin{array}{l}\text { thông tin, cũng như trích rút thông tin hố trợ cho các } \\
\text { hoạt động kinh doanh }\end{array}$ & - Khó khăn trong vận hành và bảo trì. \\
\hline & $\begin{array}{l}\text { - Cung cấp các công cụ tìm kiếm và báo cáo phong } \\
\text { phú }\end{array}$ & \\
\hline
\end{tabular}


Bảng 1. So sánh các nền tảng và công cụ xử lý log truy cập (tiếp theo)

\begin{tabular}{|c|c|c|}
\hline Nền tảng & Uu điểm & Nhược điểm \\
\hline $\begin{array}{l}\text { Sumo } \\
\text { Logic }\end{array}$ & $\begin{array}{l}\text { - Triển khai trên nền tảng điện toán đám mây nên } \\
\text { không đòi hỏi thiết bị chuyên dụng, cài đặt đơn giản } \\
\text { - Hỗ trợ xử lý nhiều dạng log } \\
\text { - Chi phí cài đặt thấp (theo gói dịch vụ) } \\
\text { - Hỗ trợ phát hiện \& cảnh báo các nguy cơ mất an } \\
\text { toàn thông tin }\end{array}$ & $\begin{array}{l}\text { - Khó khăn trong vận chuyển dữ liệu } \\
\text { log lên trung tâm xử lý } \\
\text { - Chi phí lớn cho đường truyền do } \\
\text { lượng log thường rất lớn. }\end{array}$ \\
\hline $\begin{array}{l}\text { VNCS } \\
\text { Web } \\
\text { monitoring }\end{array}$ & $\begin{array}{l}\text { - Hỗ trợ thu thập, xử lý các dạng Web log } \\
\text { - Hỗ trợ giám sát tập trung hoạt động của các } \\
\text { Website } \\
\text { - Hỗ trợ phát hiện tấn công cơ bản vào các Website, } \\
\text { bao gồm SQLi, XSS và tân công thay đổi giao diện }\end{array}$ & $\begin{array}{l}\text { - Khó khăn trong vận chuyển dữ liệu } \\
\text { log với khối lượng lớn lên trung tâm } \\
\text { xử lý đặt ở xa vị trí thu thập log } \\
\text { - Chỉ có khả năng xử lý và phân tích } \\
\text { Web log }\end{array}$ \\
\hline Logstash & $\begin{array}{l}\text { - Mã mở, miễn phí } \\
\text { - Chi phí cài đặt, vận hành thấp } \\
\text { - Hỗ trợ trích xuât các mẫu hành vi truy cập phục vụ } \\
\text { cho tối ưu hóa các trang Web }\end{array}$ & $\begin{array}{l}\text { - Khó khăn trong triển khai và bảo trì } \\
\text { do Logstash phụ thuộc vào các bộ } \\
\text { công cụ khác, gồm ElasticSearch cho } \\
\text { tìm kiếm và Kibana cho hiển thị kết } \\
\text { quả. }\end{array}$ \\
\hline Graylog & $\begin{array}{l}\text { - Mã mở, miễn phí } \\
\text { - Hỗ trợ phân tích log truy cập từ nhiều nguồn và } \\
\text { phân tích hành vi người dùng dùng cho cho phát hiện } \\
\text { và cảnh báo các truy cập bất thường cũng như trích } \\
\text { xuất các mẫu hành vi truy cập phục vụ cho tối ưu hóa } \\
\text { các trang Web }\end{array}$ & $\begin{array}{l}\text { - Không có khả năng phân tích } \\
\text { chuyên sâu các nguy cơ mất an toàn } \\
\text { thông tin, như dấu hiệu xuất hiện các } \\
\text { dạng mã độc và các dạng tấn công } \\
\text { lên các dịch vụ và tài nguyên mạng. }\end{array}$ \\
\hline LOGalyze & $\begin{array}{l}\text { - Mã mở, miễn phí } \\
\text { - Cho phép quản lý log và giám sát mạng tập trung } \\
\text { - Hỗ trợ xử lý log từ nhiều nền tảng } \\
\text { - Hỗ trợ phát hiện bất thường, sự cố theo thời gian } \\
\text { thự }\end{array}$ & $\begin{array}{l}\text { - Không có khả năng phân tích } \\
\text { chuyên sâu các nguy cơ mất an toàn } \\
\text { thông tin, như dấu hiệu xuất hiện các } \\
\text { dạng mã độc và các dạng tấn công } \\
\text { lên các dịch vụ và tài nguyên mạng. }\end{array}$ \\
\hline Webalizer & $\begin{array}{l}\text { - Mã mở, miễn phí } \\
\text { - Có khả năng phân tích nhiều dạng Web log } \\
\text { - Các báo cáo dưới dạng biểu đồ có tính biểu biễn } \\
\text { cao. }\end{array}$ & $\begin{array}{l}\text { - Chỉ có khả năng phân tích tình hình } \\
\text { sử dụng các trang Web } \\
\text { - Ít có khả năng trích xuất các thông } \\
\text { tin cho cảnh báo các nguy cơ mất an } \\
\text { toàn thông tin. }\end{array}$ \\
\hline
\end{tabular}

\section{CÁC KỸ THUẬT XỬ LÝ LOG TRUY CẬP CHO PHÁT HIỆn CÁC NGUY CƠ MẤT AN TOÀN THÔNG TIN}

Có nhiều nghiên cứu lý thuyết về phát hiện bất thường và nguy cơ mất an toàn thông tin dựa trên xử lý, phân tích log truy cập được công bố trên các tạp chí và hội thảo khoa học chuyên ngành trong khoảng hơn một thập kỷ vừa qua. Như đã nêu trong Mục 1 , bài báo này tập trung khảo sát các nghiên cứu ứng dụng phân tích log truy cập 2 dịch vụ mạng chủ chốt bao gồm dịch vụ Web và dịch vụ DNS trong phát hiện bất thường và 
các nguy cơ mất an toàn thông tin, như các dạng mã độc và tấn công, xâm nhập. Có thể chia các nghiên cứu này thành 2 nhóm chính: (i) Phát hiện botnet dựa trên phân tích log truy cập hệ thống tên miền DNS và (ii) Phát hiện bất thường và xâm nhập máy chủ $\mathrm{Web}$ dựa trên phân tích Web log. Phần tiếp theo sẽ phân tích chi tiết hơn về các nghiên cứu tiêu biểu thuộc các nhóm này.

\subsection{Phát hiện botnet dựa trên phân tích log truy cập hệ thống tên miền DNS}

Các giải pháp đề xuất phát hiện các dạng mã độc nói chung và đặc biệt là các dạng botnet nói riêng dựa trên thu thập và phân tích log truy cập hệ thống tên miền DNS được công bố khá phong phú. Trong đó, Ramachandran, Feamster, và Dagon (2006) đề xuất các kỹ thuật bộ đếm thông minh (counter-intelligence) để phát hiện các thành viên của mạng botnet thông qua việc giám sát các truy vấn cơ sở dữ liệu danh sách đen truy vấn DNS (DNSBL) từ các chủ botnet (botmaster) để xác định xem các bot (máy tính ma hay máy tính bị điều khiển) thành viên có trong danh sách đen gửi thư rác hay không. Kết quả nghiên cứu cho thấy các kỹ thuật đề xuất có khả năng phát hiện chính xác các bot thành viên tham gia gửi thư rác của mạng botnet.

Một hướng nghiên cứu phát hiện các botnet được quan tâm là phát hiện botnet thông qua việc giám sát các truy vấn từ các bot gửi hệ thống DNS để tìm máy chủ cấp lệnh và điều khiển (Command and control servers hay $C \& C$ servers). Các máy chủ C\&C được botmaster tạo lập để chuyển các lệnh và thông tin cho các bot hoạt động. Để tránh việc bị phát hiện và đưa vào danh sách đen, botmaster thường sử dụng các thuật toán để tạo ra các tên miền động cho các máy chủ $\mathrm{C} \& \mathrm{C}$. Các bot trong mạng botnet sử dụng dịch vụ DNS động (Dynamic DNS) để tìm địa chỉ IP của máy chủ C\&C để tải lệnh và mã cập nhật cho mình. Bằng việc giám sát các yêu cầu truy vấn hệ thống DNS, có thể phát hiện sự tồn tại của các bot và mạng botnet. Theo hướng này, Villamari và Brustoloni (2008) đề xuất các kỹ thuật nhận dạng botnet dựa trên phát hiện bất thường thông qua việc giám sát các truy vấn hệ thống DNS để phát hiện các truy vấn một số tên miền với tần suất rất cao và các truy vấn các tên miền không tồn tại. Điều này là khả thi do có một số lượng rất lớn các bot cùng truy vấn dịch vụ $\mathrm{DNS}$ để tìm máy chủ $\mathrm{C} \& \mathrm{C}$ và trong số đó nhiều bot chưa được cập nhật vẫn truy vấn các tên miền đã bị hủy. Mở rộng hơn, Perdisci, Corona, Dagon, và Lee (2009) đề xuất phương pháp giám sát các truy vấn DNS đệ quy để phát hiện các dịch vụ độc hại có liên quan đến các máy tính bị điều khiển của botnet, như blog rác, tin nhắn rác và thư rác. Các kết quả thử nghiệm trên hai mạng IPS thực tế trong 45 ngày cho thấy phương pháp đề xuất phát hiện chính xác các các dịch vụ độc hại.

Liên quan đến việc giám sát các truy vấn hệ thống DNS cho phát hiện botnet, một số công bố đề xuất các kỹ thuật phát hiện các tên miền được tạo ra tự động sử dụng các thuật toán, hoặc tên miền được mã độc sử dụng. Theo đó, Yadav, Reddy, và Ranjan (2010) đề xuất phương pháp phân biệt các tên miền sinh tự động bằng thuật toán thường được sử dụng trong các botnet với tên miền hợp lệ dựa trên phân tích sự phân bố các ký tự trong tên miền. Cũng với mục đích tương tự, Stalmans và Irwin (2011) sử dụng 
phương pháp cây quyết định C5.0 và thống kê Baysian để phân loại các tên miền sinh tự động với các tên hợp lệ. Cũng nhằm mục đích phát hiện các tên miền có liên quan đến mã độc botnet, Antonakakis, Perdisci, Lee, Vasiloglou, và Dagon (2011) đề xuất hệ thống phát hiện mới có tên là Kopis. Kopis thực hiện giám sát các truy vấn DNS ở mức cao trong hệ thống phân cấp DNS và phân tích các mẫu phân giải các câu truy vấn DNS toàn cục để phát hiện các tên miền có liên quan đến mã độc. Các kết quả thử nghiệm Kopis cho thấy hệ thống đạt độ chính xác đến hơn $98 \%$, tỷ lệ phát hiện sai dưới $0.5 \%$. Kopis cũng có khả năng phát hiện sớm các các tên miền có liên quan đến mã độc nhiều ngày trước khi chúng được đưa vào các danh sách đen công cộng.

Với cách tiếp cận khác, Bilge, Kirda, Kruegel, và Balduzzi (2011) giới thiệu hệ thống EXPOSURE cho phép giám sát lưu lượng truy vấn DNS trên diện rộng để phát hiện các tên miền có liên quan đến các hành vi độc hại. EXPOSURE sử dụng 15 thuộc tính của tên miền để phân biệt các tên miền đáng ngờ với tên miền hợp lệ. Các kết quả thử nghiệm cho thấy hệ thống có khả năng mở rộng tốt và nhận dạng được các tên miền mới có liên quan đến các hành vi độc hại, như chúng được sử dụng cho máy chủ $C \& C$, cho gửi thư rác và sử dụng cho các Website lừa đảo. Ngược lại, Kheir, Tran, Caron, và Deschamps (2014) đề xuất hệ thống Mentor cho phép loại bỏ các tên miền hợp lệ ra khỏi danh sách đen các tên miền của các máy chủ $\mathrm{C} \& \mathrm{C}$ sử dụng hệ thống dựa trên danh tiếng DNS tích cưc (positive DNS reputation system). Mentor sử dụng một hệ thống thu thập thông tin và các đặc tính thống kê về nội dung trang Web và tên miền để phân loại tên miền hợp lệ và tên miền đáng ngờ dựa trên học có giám sát. Các kết quả thực nghiệm trên một số danh sách đen tên miền botnet cho thấy hệ thống có khả năng nhận dạng chính xác các tên miền hợp lệ với tỷ lệ sai rất thấp.

\subsection{Phát hiện bất thường và xâm nhập máy chủ Web theo phân tích Web log}

Giám sát, thu thập và phân tích log truy cập các trang Web cho phát hiện các bất thường và các nguy cơ mất an toàn thông tin, như các dạng tấn công thường gặp lên các ứng dụng Web, gồm tấn công chèn mã SQL, tấn công XSS, tấn công thay đồi giao diện, các dạng tấn công trên diện rộng DoS/DDoS là hướng nghiên cứu được quan tâm. Meyer (2008) thuộc viện SANS, Hoa Kỳ đã tổng hợp các dạng tấn công thường gặp lên các ứng dụng Web và các kỹ thuật phát hiện tấn công lên các ứng dụng Web dựa trên phân tích Web log. Meyer (2008) cho rằng có thể sử dụng các kỹ thuật phát hiện dựa trên chữ ký, hoặc phát hiện dựa trên bất thường, hoặc kết hợp. Đồng thời, tác giả cũng phân tích các khó khăn, thách thức đối với vấn đề phát hiện tấn công lên các ứng dụng Web dựa trên phân tích Web log.

Các kỹ thuật phát hiện tấn công vào các ứng dụng Web dựa trên bất thường cũng nhận được nhiều quan tâm của cộng đồng nghiên cứu. Khác với kỹ thuật phát hiện dựa trên chữ ký, phát hiện dựa trên bất thường có khả năng phát hiện các dạng tấn công mới do nó không yêu cầu phải có trước thông tin về các dạng tấn công, xâm nhập. Theo đó, Kruegel và Giovanni (2003) đề xuất phương pháp phát hiện tấn công vào các ứng dụng Web dựa trên bất thường thông qua việc giám sát và phân tích các tham số trong yêu 
cầu máy khách gửi đến máy chủ Web. Hệ thống tự động học để xây dựng hồ sơ phát hiện cho từng ứng dụng Web thông qua việc phân tích các thuộc tính của yêu cầu truy cập, như độ dài, phân bố các ký tự, trật tự các tham số,... Hệ thống có khả năng phát hiện các dạng tấn công mới do không yêu cầu phải biết trước các thông tin về chúng và có thể giảm thời triển khai do việc xây dựng hồ sơ phát hiện được thực hiện tự động. Tương tự, Cho và Cha (2004) giới thiệu SAD, một hệ thống phát hiện các tấn công vào dịch vụ Web dựa trên phát hiện bất thường trong phiên làm việc Web. SAD xây dựng hồ sơ mô hình hóa phiên làm việc Web bình thường sử dụng phương pháp ước lượng tham số Baysian. Sau đó $\mathrm{SAD}$ giám sát thu thập Web log, trích xuất các phiên và so sánh với hồ sơ đã xây dựng để phát hiện các bất thường. Kết quả thử nghiệm phát hiện tấn công mô phỏng cho thấy SAD có khả năng phát hiện vượt trội so với Snort (2017), một hệ thống phát hiện xâm nhập dựa trên chữ ký mã mở được sử dụng rất rộng rãi.

Ứng dụng các kỹ thuật như lập trình Gen và học máy trong phân tích Web log cho phát hiện bất thường và các dạng tấn công lên ứng dụng Web cũng là hướng nghiên cứu được quan tâm. Tiêu biểu, Faradzhullaev (2008) đề xuất ứng dụng nhiều phương pháp, bao gồm xác suất thống kê và lập trình Gen để xử lý và phân tích Web log cho phát hiện bất thường. Các kết quả thử nghiệm phân tích Web log của máy chủ Web cho thấy, phương pháp đề xuất có khả năng phát hiện chính xác các truy vấn bất thường đến máy chủ Web từ người dùng ác tính. Mở rộng hơn, Moh, Pininti, Doddapaneni, và Moh (2016) đề xuất phương pháp phát hiện tấn công ứng dụng Web dựa trên phân tích Web $\log$ sử dụng kết hợp giữa đối sánh mẫu và học máy Bayes Net. Kết quả thử nghiệm trên 10,000 bản ghi Web log cho thấy phương pháp kết hợp đề xuất có khả năng nâng cao đáng kể độ chính xác phát hiện tấn công chèn mã SQL.

Mặc dù không có khả năng phát hiện các dạng tấn công mới, hoặc chưa biết, các kỹ thuật phát hiện dựa trên chữ ký vẫn được sử dụng rộng rãi do khả năng phát hiện nhanh và chính xác các dạng tấn công đã biết. Tiêu biểu, Yen và ctg. (2013) đề xuất Beehive, một hệ thống phân tích log cỡ lớn dựa trên chữ ký nhằm phát hiện các hành vi đáng ngờ trong các mạng doanh nghiệp. Beehive có khả năng khai phá tự động khối lượng lớn dữ liệu log do các thiết bị đảm bảo an ninh tạo ra và gửi các cảnh báo về các sự kiện đáng ngờ cho đội phân tích an toàn thông tin thực hiện phân tích chuyên sâu. Kết quả thử nghiệm cho thấy Beehive có khả năng nhận dạng nhiều hành vi đáng ngờ mà các thiết bị và phần mềm an ninh không phát hiện được.

Tiền xử lý và chuyển đổi định dạng dữ liệu về định dạng thuận tiện cho phân tích $\log$, đặc biệt là với hệ thống hỗ trợ xử lý nhiều định dạng log khác nhau cũng là chủ đề được quan tâm. Tiêu biểu, Salama, Marie, El-fangary, và Helmy (2011) đề xuất một thủ tục tiền xử lý giảm nhiễu và chuyển đổi Web log từ nhiều định dạng sinh bởi các máy chủ Web khác nhau về định dạng XML (eXtensible Markup Language) thống nhất để thuật tiện cho việc truy vết và phát hiện các dạng tấn công, xâm nhập lên ứng dụng Web. Các tác giả cũng chỉ rõ sự khác biệt giữa tiền xử lý log cho phát hiện xâm nhập và tiền xử lý log cho khai phá hành vi sử dụng Web. 


\section{NHẬN XÉT VÀ ĐỀ XUẤT}

\subsection{Nhận xét}

Có thể thấy, các nền tảng và công cụ xử lý, phân tích log hiện có khá phong phú, có khả năng xử lý nhiều dạng log, đồng thời cung cấp nhiều tính năng đa dạng. Tuy nhiên, một số công cụ mạnh có khả năng phân tích, nhận dạng các hành vi truy cập bất thường và cảnh báo các nguy cơ mất an toàn thông tin thường có chi phí cài đặt và vận hành lớn (như IBM QRadar SIEM, Splunk, VNCS web monitoring), phù hợp với các cơ quan, tổ chức có quy mô hệ thống mạng và nguồn lực lớn, như các cơ quan chính phủ trung ương, các tổ chức tài chính, ngân hàng. Tuy nhiên, chúng không thực sự phù hợp với các cơ quan, tổ chức có quy mô hệ thống mạng và nguồn lực cỡ vừa và nhỏ. Một số nền tảng và công cụ khác có chi phí cài đặt và vận hành thấp hơn, nhưng tính năng hạn chế (chỉ hỗ trợ phân tích Web log và trích xuất các báo cáo truy cập như Webalizer) và đặc biệt không hỗ trợ phân tích chuyên sâu, như phân tích phát hiện dấu hiệu xuất hiện của các dạng mã độc và các dạng tấn công, xâm nhập hệ thống và mạng (như Graylog, LOGalyze). Các nền tảng và công cụ xử lý, phân tích log thuộc dạng này cũng không phù hợp với yêu cầu phân tích log truy cập phục vụ phát hiện các truy cập bất thường và các nguy cơ mất an toàn thông tin, như dấu hiện của các dạng mã độc và các dạng tấn công, xâm nhập trong hệ thống mạng của các cơ quan, tổ chức.

Các nghiên cứu về phát hiện bất thường và các nguy cơ mất an toàn thông tin, như các dạng tấn công, xâm nhập và mã độc (như botnet) dựa trên xử lý và phân tích $\log$ truy nhập khá phong phú, với nhiều công bố trên các tạp chí và kỷ yếu hội thảo khoa học quốc tế. Tuy nhiên, hầu hết các đề xuất chỉ xem xét từng vấn đề (phát hiện bất thường, hoặc các nguy cơ mất an toàn thông tin) một cách riêng rẽ. Hơn nữa, các hệ thống đề xuất thường chỉ xử lý một dạng xác định log truy cập (DNS log, hoặc Web $\log$ ) và chưa đặt vấn đề xử lý tích hợp nhiều dạng log thu thập từ nhiều nguồn phục vụ phát hiện các truy cập bất thường và các nguy cơ mất an toàn thông tin.

\section{2. Đề xuất}

Từ các phân tích nêu trên có thể thấy, với các cơ quan, tổ chức có quy mô hệ thống mạng và nguồn lực cỡ vừa và nhỏ, không có nền tảng, công cụ xử lý, phân tích $\log$ sẵn có thực sự phù hợp, trong đó yêu cầu hỗ trợ thu thập, xử lý phân tích log từ nhiều dạng $\log$, từ nhiều nguồn, đa nền tảng và cho phép phát hiện bất thường và các nguy cơ mất an toàn thông tin. Hướng giải quyết của nhóm nghiên cứu là đề xuất phát triển một hệ thống dựa trên xử lý, phân tích log truy cập tích hợp và các nền tảng mã nguồn mở hỗ trợ phát hiện bất thường và các nguy cơ mất an toàn thông tin cho hệ thống mạng của các cơ quan, tổ chức có quy mô và nguồn lực hạn chế. Các nền tảng và công cụ mã nguồn mở được tận dụng khai thác tối đa, nhằm giảm chi phí phát triển hệ thống. Kiến trúc tổng thể của hệ thống đề xuất được mô tả trên Hình 9. 
(1)

Thu thập và tiền xử lý log
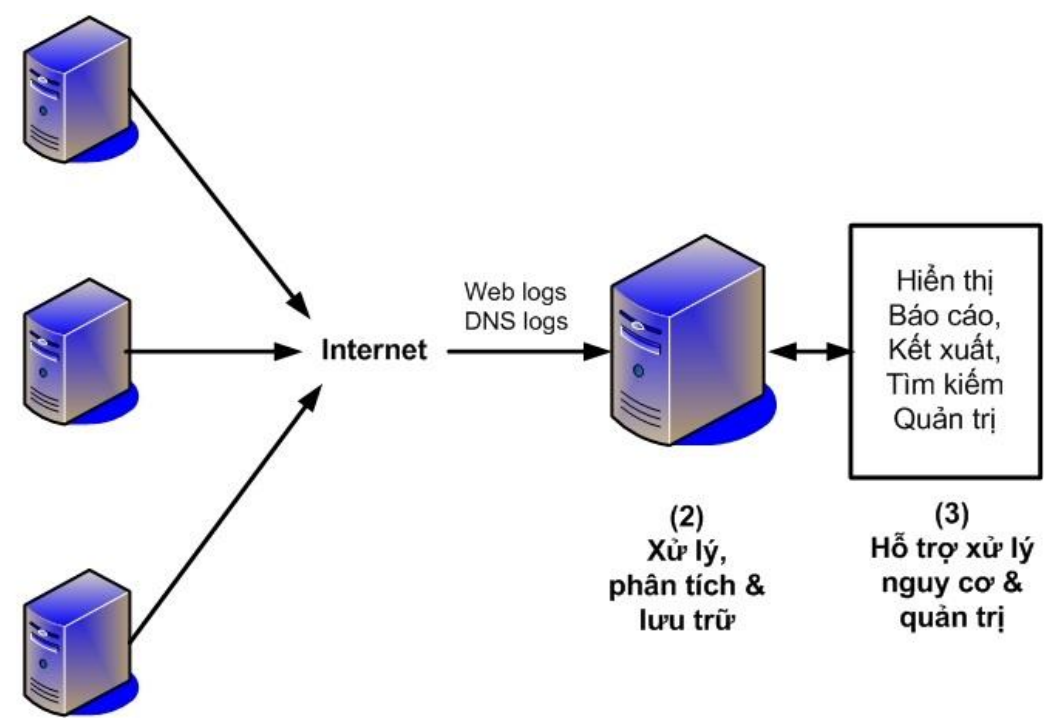

(2)

Xử lý, phân tích \& lưu trữ
(3)

Hỗ trợ xử lý nguy $\operatorname{co} \&$ quản trị

\section{Hình 9. Mô hình kiến trúc đề xuất cho hệ thống phát hiện bất thường và các nguy cơ mất an toàn thông tin dựa trên xử lý log truy cập}

Theo đó, hệ thống được hình thành từ 3 phân hệ: (i) Phân hệ thu thập và tiền xử lý $\log$ có nhiệm vụ thu thập DNS và Web logs từ các hệ thống máy chủ dịch vụ, thực hiện tiền xử lý, chuẩn hóa và vận chuyển trực tiếp về phân hệ xử lý trung tâm thông qua mạng Internet. Các thành phần của phân hệ này được cài đặt phân tán ở hệ thống mạng của cơ quan, tổ chức; (ii) Phân hệ xử lý, phân tích và lưu trữ log có nhiệm vụ xử lý, phân tích và quản lý log sau chuẩn hóa nhằm trích xuất các thông tin có giá trị hỗ trợ phát hiện các hành vi bất thường và cảnh báo các nguy cơ mất an toàn thông tin trong hệ thống mạng của cơ quan, tổ chức; và (iii) Phân hệ hỗ trợ xử lý nguy cơ an toàn thông tin và quản trị có nhiệm vụ cung cấp các công cụ hiển thị, báo cáo, tìm kiếm kết quả phân tích và các công cụ cảnh báo đến người quản trị. Giao diện là dựa trên nền Web, hỗ trợ phân quyền người dùng và nhóm người dùng.

\section{KẾT LUẬN}

Giám sát, phát hiện bất thường và các nguy cơ mất an toàn thông tin cho hệ thống các máy chủ dịch vụ là một trong các giải pháp quan trọng trong lớp các giải pháp của mô hình đảm bảo an toàn thông tin "defense in depth" (phòng vệ theo chiều sâu, hay phòng vệ nhiều lớp) cho hệ thống mạng. Bài báo này khảo sát một số nền tảng, công cụ và kỹ thuật xử lý, phân tích log truy cập dịch vụ phục vụ phát hiện bất thường và các nguy cơ mất an toàn thông tin. Trên cơ sở khảo sát, đánh giá về sự phù hợp của các nền tảng, công cụ và kỹ thuật đã có, chúng tôi đề xuất phát triển hệ thống dựa trên xử lý, phân tích log truy cập tích hợp và các nền tảng mã mở hỗ trợ phát hiện bất thường và các nguy cơ mất an toàn thông tin cho các cơ quan, tổ chức có quy mô hệ thống mạng và nguồn lực hạn chế. Hướng tiếp cận này hỗ trợ phát hiện bất thường và các nguy cơ mất an toàn thông tin, đồng thời có thể giúp giảm chi phí phát triển hệ thống nhờ khai thác các nền tảng và công cụ mã mở. Hướng nghiên cứu tiếp theo của bài báo là phát 
triển thành đề tài nghiên cứu, phát triển hệ thống giám sát phát hiện bất thường và các nguy cơ mất an toàn thông tin dựa trên phân tích log truy cập, ứng dụng cho các hệ thống dịch vụ công cấp tỉnh, thành phố.

\section{LỜI CẢM ƠN}

Các tác giả bài báo chân thành cảm ơn sự hỗ trợ nhân lực của Lab An toàn Thông tin, Học viện Công nghệ Bưu chính Viễn thông, Hà Nội và sự hỗ trợ tài chính từ đề tài KC.01.05/16-20, Chương trình khoa học và công nghệ trọng điểm cấp quốc gia giai đoạn 2016-2020, Bộ Khoa học và Công nghệ.

\section{TÀI LIỆU THAM KHẢO}

Antonakakis, M., Perdisci, R., Lee, W., Vasiloglou, N. I. I., \& Dagon, D. (2011). Detecting malware domains at the upper DNS hierarchy. Retrieved from http://astrolavos.gatech.edu/articles/kopis.pdf.

Bilge, L., Kirda, E., Kruegel, C., \& Balduzzi, M. (2011). EXPOSURE: Finding malicious domains using passive DNS analysis. Paper presented at The Network and Distributed System Security Symposium, USA.

Cho, S., \& Cha, S. (2004). SAD: Web session anomaly detection based on parameter estimation. Journal of Computers \& Security, 23(4), 312-319.

Cục An toàn Thông tin. (2016). Báo cáo An toàn thông tin Việt Nam 2016. Hà Nội, Việt Nam: Cục An toàn Thông tin.

Faradzhullaev, R. (2008). Analysis of Web server log files and attack detection. Journal of Automatic Control and Computer Sciences, 42(1), 50-54.

Graylog. (2017). Enterprise log management for all. Retrieved from https://www. graylog.org.

IBM QRadar. (2017). How QRadar SIEM can help your business. Retrieved from https://www.ibm.com/ms-en/marketplace/ibm-qradar-siem.

Jiang, N., Cao, J., Jin, Y., Li, L., \& Zhang, Z. L. (2010). Identifying suspicious activities through DNS failure graph analysis. Paper presented at The IEEE International Conference on Network Protocols (ICNP), Japan.

Kheir, N., Tran, F., Caron, P., \& Deschamps, N. (2014). Mentor: Positive DNS reputation to skim-off benign domains in botnet $C \& C$ blacklists. Paper presented at The ICT Systems Security and Privacy Protection Conference, Morocco.

Kruegel, C., \& Giovanni, V. (2003). Anomaly detection of Web-based attacks. Paper presented at The ACM Conference on Computer and Communications Security, USA. 
LOGalyze. (2017). Free unlimited log management tool for everyone. Retrieved from http://www.logalyze.com.

Logstash. (2017). Centralize, transform, \& stash your data. Retrieved from http://logstash.net.

Ma, X., Zhang, J., Li, Z., Li, J., Tao, J., Guan, X., Lui, J. C., \& Towsley, D. (2015). Accurate DNS query characteristics estimation via active probing. Journal of Network Computing Applications, 47, 72-84.

Meyer, R. (2008). Detecting attacks on Web applications from log files. Retrieved from https://www.sans.org/reading-room/whitepapers/logging/detecting-attacks-webapplications-log-files-2074.

Moh, M., Pininti, S., Doddapaneni, S., \& Moh, T. S. (2016). Detecting Web attacks using multi-stage log analysis. Paper presented at The International Conference on Advanced Computing (IACC), India.

Perdisci, R., Corona, I., Dagon, D., \& Lee, W. (2009). Detecting malicious flux service networks through passive analysis of recursive DNS traces. Paper presented at The Annual Computer Security Applications Conference (ACSAC), USA.

Ramachandran, A., Feamster, N., \& Dagon, D. (2006). Revealing botnet membership using DNSBL counter-intelligence. Retrieved from https://www.usenix.org/ legacy/event/sruti06/tech/full_papers/ramachandran/ramachandran_html/index.h tml.

Salama, S. E., Marie, M. I., El-fangary, L. M., \& Helmy, Y. K. (2011). Web server logs preprocessing for Web intrusion detection. Journal of Computer and Information Science, 4(4), 123-133.

Snort. (2017). Snort IDS. Retrieved from http://www.snort.org.

Splunk. (2017). Hyatt innovates and ensures seamless customer experience with Splunk. Retrieved from http://www.splunk.com.

Stalmans, E., \& Irwin, B. (2011). A framework for DNS based detection and mitigation of malware infections on a network. Paper presented at The Information Security South Africa (ISSA), South Africa.

Sumo Logic. (2017). Delight your customers with modern analytics. Retrieved from http://www.sumologic.com.

Villamari, S. R., \& Brustoloni, J. C. (2008). Identifying botnets using anomaly detection techniques applied to DNS traffic. Paper presented at The Consumer Communications and Networking Conference (CCNC), USA.

VNCS. (2017). Giải pháp giám sát Website tập trung. Retrieved from http://vncs.vn/ portfolio/giai-phap-giam-sat-websites-tap-trung.

Webalizer. (2017). The Webalizer. Retrieved from http://www.webalizer.org. 
Yadav, S., Reddy, A. K. K., Reddy, A., \& Ranjan, S. (2010). Detecting algorithmically generated malicious domain names. Paper presented at The ACM SIGCOMM Conference on Internet Measurement, Australia.

Yen, T. F., Oprea, A., Onarlioglu, K., Leetham, T., Robertson, W., Juels, A., \& Kirda, E. (2013). Beehive: Large-scale log analysis detecting suspicious activity in enterprise networks. Paper presented at The Annual Computer Security Applications Conference, USA. 